\title{
Optic Nerve Head Microcirculation in Eyes With Vogt-Koyanagi-Harada Disease Accompanied by Anterior Ischemic Optic Neuropathy: a Case Report
}

\section{Yui Yamashita}

Faculty of Medicine and Graduate School of Medicine, Hokkaido University

\section{Yuki Hashimoto}

Faculty of Medicine and Graduate School of Medicine, Hokkaido University

Kenichi Namba ( $\sim$ knamba@med.hokudai.ac.jp)

Faculty of Medicine and Graduate School of Medicine, Hokkaido University https://orcid.org/00000002-5016-851X

\section{Kazuomi Mizuuchi}

Faculty of Medicine and Graduate School of Medicine, Hokkaido University

\section{Susumu Ishida}

Faculy of Medicine and Graduate School of Medicine, Hokkaido University

\section{Short Report}

Keywords: Anterior ischemic optic neuropathy, Laser speckle flowgraphy, Optical coherence tomography, Optic nerve head microcirculation, Vogt-Koyanagi-Harada (VKH) disease

Posted Date: March 16th, 2021

DOl: https://doi.org/10.21203/rs.3.rs-292547/v1

License: (9) This work is licensed under a Creative Commons Attribution 4.0 International License. Read Full License 


\section{Abstract}

Purpose: It has been reported that anterior ischemic optic neuropathy (AION) is an infrequent complication of Vogt-Koyanagi-Harada (VKH) disease; however, the physiological changes have not been understood. We quantitatively examined sequential changes in the morphology and circulation hemodynamics using an optical coherence tomography (OCT) C-scan and laser speckle flowgraphy (LSFG) in a patient with VKH disease accompanied by AION.

Case presentation: A 65-year-old female complained of blurred vision in both of her eyes. She presented with optic disc swelling and remarkable choroidal thickening detected by OCT bilaterally. Indocyanine green angiography in the middle phase showed multiple hypofluorescent dark dots scattering around the fundus. With the use of Goldmann perimetry, bilateral visual field defects were detected; these were similar to those of inferior altitudinal hemianopsia. Pleocytosis was detected. The patient was diagnosed with VKH disease, suspected to be accompanied by AION in both eyes. She received methylprednisolone pulse therapy followed by oral prednisolone. With these treatments, optic disc swelling disappeared; however, optic disc atrophy with visual field defects remained in both eyes. An OCT C-scan showed the ganglion cell complex (GCC) and circumpapillary retinal nerve fiber layer (cpRNFL) thickness getting thinner below the normal range, and LSFG showed a decrease in optic nerve head tissue microcirculation during follow-up. These results supported the occurrence of AION in this patient with VKH disease.

Conclusion: The analyses of GCC and cpRNFL thicknesses with an OCT C-scan and optic nerve head microcirculation with LSFG would be useful for supporting the occurrence of AION in cases of VKH disease.

\section{Introduction}

Vogt-Koyanagi-Harada (VKH) disease is a systemic, immune-mediated, inflammatory condition that is considered to be caused by autoimmunity against melanocytes [1]. Acute symptoms at the initial onset include bilateral granulomatous intraocular inflammation, meningitis, and sensorineural hearing loss. Intraocular involvements are characterized by severe posterior segment inflammation, including serous retinal detachment (SRD) and optic disc swelling, whereas anterior segment inflammation is either absent or mild [1, 2].

Previously, a few cases were reported in which severe visual field defects were seen with disc swelling, indicating that anterior ischemic optic neuropathy (AION) had developed at the onset of VKH disease [3, 4]. Nakao et al. reported 6 patients who showed visual field defects with the retinal nerve fiber layer (RNFL) thickness decrease, suggesting the presence of AION among 15 patients with disc swelling out of $52 \mathrm{VKH}$ disease patients [5].

Optical coherence tomography (OCT) is a useful tool for visualizing the retinal layer and choroidal layer. In VKH disease, choroidal thickness detected by OCT is considered to be a valuable index of disease activity. With active inflammation the choroid is thickened, and it is decreased (along with the resolution 
of the inflammation) with the administration of the treatment [6]. In addition, OCT can segment the layers of the retina and choroid. It has already been shown that the thinning of the RNFL and the ganglion cell complex (GCC) are characteristic findings seen in optic nerve atrophy, including AION [5, 7].

Laser speckle flowgraphy (LSFG) is a non-invasive imaging modality capable of quantitatively evaluating ocular blood flow velocity. LSFG targets moving erythrocytes that produce blurring within the speckle pattern using a diode laser at the wavelength of $830 \mathrm{~nm}$ to illuminate the ocular fundus. The mean blur rate (MBR), automatically calculated from variations in the degree of blurring, is a quantitative index of the relative blood flow velocity. The measurement results have high reproducibility [8]. LSFG is a useful tool for detecting choroidal circulation changes due to inflammation in patients with VKH disease in which the macular MBR is decreased [6]. LSFG can also be used to detect ischemic optic neuropathy in which the MBR of the optic disc tissue is decreased [9].

As mentioned above, OCT and LSFG are useful tools for diagnosing and examining the clinical stage of both VKH disease and AION. This is the first known case report of VKH disease suspected to be accompanied by AION in which the clinical course could be followed up with OCT and LSFG.

\section{Case Presentation}

A 65-year-old female complained of blurred vision in her both eyes (OU), which persisted for 1 week. The patient's medical and family histories were unremarkable.

Her best-corrected visual acuity (BCVA) was 0.2 in the right eye (OD) and 0.6 in the left eye (OS) with hyperopic refractive error. A slit-lamp examination revealed no abnormal findings in the anterior segment OU. Funduscopic examination showed optic disc swelling OU, and OCT showed remarkable choroidal thickening as well as optic disc swelling OU (Fig. 1a, b, 3a). Late-phase fluorescein angiography showed slight leakages from retinal capillary vessels and optic disc staining (Fig. 1C, d). Indocyanine green angiography (IA) at the middle-phase showed multiple hypofluorescent dark dots (HDDs) scattering all around the fundus (Fig. 1e, f). Visual evoked potentials were non-recordable OU. With the use of Goldmann perimetry, bilateral visual field defects, similar to those of inferior altitudinal hemianopsia (Figs. 1g, h), were detected. The cerebrospinal fluid cell count was $11 / \mu \mathrm{l}$, indicating pleocytosis. The patient was diagnosed with VKH disease, suspected to be accompanied by AION OU.

The patient received intravenous methylprednisolone that was initially administered at $1000 \mathrm{mg} / \mathrm{day}$ for 3 consecutive days (pulse therapy). Oral prednisolone was then initiated and tapered with the following schedule: 10 days at $40 \mathrm{mg} /$ day, 10 days at $30 \mathrm{mg} /$ day, 10 days at $25 \mathrm{mg} /$ day, 1 month at $20 \mathrm{mg} /$ day, 1 month at $15 \mathrm{mg} /$ day, 1 month at $10 \mathrm{mg} /$ day, 1 month at $5 \mathrm{mg} /$ day, and $1 \mathrm{month}$ at $2.5 \mathrm{mg}$. Then, the therapy stopped.

Her optic disc swelling disappeared with the therapy; however, her optic discs became slightly pale and her visual field defects still remained OU (Fig. 2a-d). BCVA was 0.3 OD and 0.6 OS 5 months later. No recurrence has been observed so far. 


\section{Methods}

\section{Choroidal thickness measurements}

OCT (RS-3000 Advance ${ }^{\circledR}$; Nidek, Gamagori, Japan) measurements were performed OU at the initial visit, at weeks 1,2 , and 3 , and at 1, 4, 5, and 12 months after the initiation of the treatment. Central choroidal thickness (CCT) was determined by manually measuring the distance at the fovea from the outer border of the hyperreflective line corresponding to the retinal pigment epithelium to the outer border of the choroid (Fig. 3a-d), using a horizontal scan through the fovea (scan length, $12.0 \mathrm{~mm}$ ). Two authors (YY, $\mathrm{YH})$, blinded to the patient's clinical information, independently evaluated the OCT images. CCT reaching $>800 \mu \mathrm{m}$ was defined as $800 \mu \mathrm{m}$, because the inner scleral border could not be visualized with OCT.

\section{GCC and RNFL thickness measurements}

The OCT C-scan GCC thickness and circumpapillary (cp) RNFL thickness were measured the initial visit and at weeks 1, 2, and 3 as well as at 1, 4, 5, and 12 months.

GCC thickness values were automatically calculated and compared to normative database by software equipped with OCT (Fig. 4a, c, e, g). The mean GCC thickness was calculated from 8 sectors segmented around the macula $(6 \mathrm{~mm} \times 6 \mathrm{~mm})$, which excluded the fovea sector $(1 \mathrm{~mm} \times 1 \mathrm{~mm})$. The disc circle scan pattern captured an image of a circle with a $3.45 \mathrm{~mm}$ diameter around the disc that allowed cpRNFL thickness analysis, compared to those in the normative database (Fig. 4b, d, f, h). The red color zone depicted an extremely thinning area which represented an abnormal to normal database percentage of < $1 \%$. The yellow and white thickened zones represented an abnormal to normal database percentage of < $5 \%$. The green area depicted a relatively normal area, evaluated as being between $5 \%$ and $95 \%$ in a population of normal eyes.

\section{Macular and optic disc circulation measurements}

LSFG measurements using LSFG-NAVI (Softcare, Fukuoka, Japan) were performed to quantitatively examine choroidal and optic nerve head (ONH) blood flow velocity. LSFG results were examined 5 consecutive times at the initial visit and also at 1,2, and 3 weeks, as well as at 1, 4, 5, and 12 months after treatment. Information has been available online on the mechanism by which LSFG operates, along with its measurement method [10].

On the color map, a circle band was set at the macula in each eye (Fig. 5a, c, e, g) and ONH (Fig. 5b, d, f, h). Since the origin of the macular MBR is derived from the choroid (because of the macula lacking retinal vessels), the macular MBR indicates choroidal blood flow velocity. The blood circulation of ONH was evaluated with the $\mathrm{ONH}$ tissue MBR: the MBR of all ONH area minus the MBR of ONH vascular area. The positions of circle bands were determined manually as being in exactly the same place as those used at baseline by comparing the fundus photographs and the LSFG color map images. Each MBR was automatically calculated using LSFG Analyzer software (v 3.0.47; Softcare). Sequential changes in the 
average MBR were evaluated as the changing rates of the average MBR to the baseline values, as previously described, since MBR is a quantitative index of the "relative" blood flow velocity.

\section{Perfusion pressure measurements}

As previously demonstrated [11], within a certain range, the relationship between choroidal blood flow and ocular perfusion pressure (OPP) is linear in healthy subjects with normal eyes. To exclude the possibility of such physiological responses from the results, the patient's blood pressure and intraocular pressure (IOP) were measured to calculate the OPP. The mean blood pressure (BPm) was calculated from systolic blood pressure (BPs) and diastolic blood pressure (BPd) readings, according to the following equation: $B P m=B P d+1 / 3(B P s-B P d)$. OPP was calculated using the following equation: $O P P=2 / 3$ BPm - IOP.

\section{Results}

\section{CCT changes}

The marked thickening of the choroid seen at the initial visit was recovered to the normal range with the therapy within 2 weeks and maintained until 5 months later (Fig. 3).

\section{GCC and CpRNFL changes}

The GCC thickness was almost normal at the initial visit; however, it changed and was observed as being thin in the macular area OU 2 weeks later. It continued to get thinner (Fig. 4a, c, e, g).

cpRNFL thickened at the initial visit and recovered to a normal range 1 month later. However, it changed and became thin, measuring below the normal range at 5 months (Fig. $4 b, d, f, h$ ).

\section{Macular and $\mathrm{ONH}$ tissue MBR changes}

LSFG was performed in 2 areas: the macula and ONH. The MBR in the macular area was low at the initial visit and it gradually increased with the treatment OU (Fig. 5a, c, e, g). The values at the initial visit and at 1,2 , and 3 weeks, as well as at 1, 4, 5, and 12 months after the treatment were as follows: $7.2 \pm 0.4 ; 8.1 \pm$ $0.2 ; 11.3 \pm 0.5 ; 11.3 \pm 0.7 ; 11.4 \pm 0.5 ; 12.1 \pm 0.7 ; 12.0 \pm 0.5 ;$ and $13.2 \pm 1.0 \mathrm{OD} ;$ and $4.9 \pm 0.1 ; 5.7 \pm 0.2 ; 9.3$ $\pm 0.4 ; 9.6 \pm 0.1 ; 9.9 \pm 0.3 ; 10.8 \pm 0.5 ; 10.8 \pm 0.4 ;$ and $12.0 \pm 0.4 \mathrm{OS}$, respectively. When the changing rates in MBR were compared with the baseline values (100\%), $12.5 \%, 56.9 \%, 56.9 \%, 58.3 \%, 68.0 \%, 80.5 \%$, and $83.3 \%$ increments OD, and $16.3 \%, 89.7 \%, 95.9 \%, 102.0 \%, 120.4 \%, 120.4 \%$, and $144.8 \%$ increments OS were noted at 1, 2, and 3 weeks, along with 1, 4, 5, and 12 months after treatment, respectively (Fig. 5i).

Blood flow velocity at the $\mathrm{ONH}$ was evaluated with the tissue MBR. In comparison with the tissue MBR at the initial visit, it gradually decreased OU with the treatment (Fig. 5b, d, f, h). Changes in the values at the initial visit and at 1,2, and 3 weeks, as well as at 1, 4, 5, and 12 months after the treatment were as follows: $21.6 \pm 0.8 ; 16.4 \pm 0.8 ; 13.8 \pm 0.8 ; 14.2 \pm 0.8 ; 13.7 \pm 0.5 ; 11.2 \pm 0.2 ; 12.6 \pm 0.9$; and $10.7 \pm 0.6$ OD, 
and $20.5 \pm 1.5 ; 15.5 \pm 0.3 ; 14.0 \pm 0.7 ; 13.8 \pm 0.4 ; 15.0 \pm 0.6 ; 12.5 \pm 0.3 ; 11.8 \pm 0.3$; and $12.7 \pm 0.5$ OS, respectively. When the changing rates in MBR were compared with the baseline values (100\%), $24.1 \%$, $36.2 \%, 34.3 \%, 36.6 \%, 48.2 \%, 41.7 \%$, and $50.5 \%$ decrements OD, and $24.4 \%, 31.8 \%, 32.7 \%, 26.9 \%, 39.1 \%$, $42.5 \%$, and $37.9 \%$ decrements OS were noted at 1,2 , and 3 weeks, as well as at $1,4,5$, and 12 months after treatment, respectively (Fig. 5j). OPP was unaltered OU during the follow-up period.

\section{Discussion}

In this study, we quantitatively evaluated changes of the choroid and ONH blood flow velocity using LSFG and changes of the retinal layer thickness using OCT in a patient with VKH disease accompanied by AION. Findings obtained at the onset, including remarkable choroidal thickening detected by OCT and multiple HDDs seen in IA bilaterally, along with the presence of pleocytosis, confirmed the diagnosis of VKH disease. The increase of the macular MBR along with the treatment supported it. Additionally, optic atrophy seen after the treatment suggested the occurrence of AION combined with the onset of VKH disease, which were supported by our findings (the GCC and CPRNFL thinning and the ONH tissue MBR decrease during the 12-month follow-up period). This study is the first to show the quantitative changes over time in the $\mathrm{ONH}$ microcirculation in VKH disease associated with AION.

AION is not a common complication with VKH disease. Nakao et al. reported that in the consecutive series of 52 VKH disease patients, 15 (28.8\%) showed optic disc swelling; among them, 6 (11.5\%) were suspected to be accompanied with AION with the findings of visual field defects, optic disc atrophy, and the decrease of RNFL thickness, while none of the patients without optic disc swelling developed AION [5]. This result indicated that optic disc swelling is a risk factor for AION development in VKH disease patients. Actually, our patient in this report also showed severe bilateral optic disc swelling at the onset.

Usually, AION is diagnosed based on age, disc appearance, and the results of a visual field test and the RNFL thickness [5]; however, these findings do not directly indicate the ischemia of the optic nerve. LSFG is a non-invasive technique to quantitatively measure blood flow velocity, and it can support both the diagnosis and evaluation of the activity in various diseases with choroidal abnormalities, glaucoma, and AION. Maekubo et al. previously reported that with AION, the ONH tissue MBR was $29.5 \%$ lower than that of unaffected eyes [9]. In our case as well, LSFG showed a decrease in the ONH tissue MBR, strongly supporting the diagnosis of the development of AION.

The analysis of the retinal layer thickness with the OCT C-scan revealed the appearance of the thinning of the GCC layer in the macular area 2 weeks later and the thinning of the cpRNFL at 5 months, which was thickened at the onset. In AION, it is considered that the demyelination of the optic nerve results in the thinning of cpRNFL. Subsequently, it induces the apoptosis of retinal ganglion cells, resulting in the thinning of the GCC layer. Indeed, it was already reported that in AION, the GCC thickness decreased at 1 month from the onset and the cpRNFL thickness increased at the onset and decreased 6 months later [7]. Results of the retinal layer thickness analysis in our case were consistent with changes reported in AION, ensuring the complication of AION with VKH disease. 
The incidence of AION associated with VKH disease has been reported to be 11.5\%; however, the diagnosis of AION was made based on the disc appearance and results of the visual field test, both of which were subjective examinations, together with $\mathrm{cpRNFL}$ thickness [5]. Further studies with a larger number of cases involving an OCT C-scan and LSFG may reveal the exact incidence of AION in VKH disease.

\section{Conclusion}

The analyses of retinal layer thickness with an OCT C-scan and ONH microcirculation with LSFG could support the diagnosis of AION in cases of VKH disease. These examinations would be essential in not only the diagnosis but also observation of AION complicated with VKH disease.

\section{Abbreviations}

VKH: Vogt-Koyanagi-Harada; SRD: serous retinal detachment; AION: anterior ischemic optic neuropathy; RNFL: retinal nerve fiber layer; OCT: optical coherence tomography; GCC: ganglion cell complex; LSFG: laser speckle flowgraphy; MBR: mean blur rate; OU: both eyes; BCVA: best-corrected visual acuity; OD: right eye; OS: left eye; IA: indocyanine green angiography; HDDs: hypofluorescent dark dots; CCT: central choroidal thickness; $c \mathrm{c}$ : circumpapillary; ONH: optic nerve head; OPP: ocular perfusion pressure; IOP: intraocular pressure; BPm: mean blood pressure; BPs: systolic blood pressure; BPd: diastolic blood pressure

\section{Declarations}

\section{Ethics approval and consent to participate}

Not required

\section{Consent for pablication}

Informed consent was obtained in writing from the patient and his parent for the use of the patient's information for the purpose of this report.

\section{Availability of data and material}

Not Applicable.

\section{Competing interests}

The following authors declare that they have no competing interests: YY, YH, KN, KM, and SI.

\section{Funding}

No funding or grant support. 


\section{Authors' contributions}

All authors read and approved the final manuscript.

\section{Acknowledgements}

Not applicable.

\section{References}

1. Moorthy RS, Inomata H, Rao NA (1995) Vogt-Koyanagi-Harada syndrome. Surv Ophthalmol 39: 265292

2. Sugiura S (1978) Vogt-Koyanagi-Harada disease. Jpn J Ophthalmol 22: 9-35

3. Yokoyama A, Ohta K, Kojima H, Yoshimura N (1999) Vogt-Koyanagi-Harada disease masquerading anterior ischaemic optic neuropathy. $\mathrm{Br} \mathrm{J}$ Ophthalmol 83: 123

4. Abematsu N, Shimonagano Y, Nakao K, Sakamoto T, Shimizu K, Hirashima S (2006) A case of anterior ischemic optic neuropathy associated with Vogt-Koyanagi-Harada disease. Nippon Ganka Gakkai Zasshi 110: 601-606

5. Nakao K, Mizushima Y, Abematsu N, Goh N, Sakamoto T (2009) Anterior ischemic optic neuropathy associated with Vogt-Koyanagi-Harada disease. Graefes Arch Clin Exp Ophthalmol 247: 1417-1425

6. Hirooka K, Saito W, Namba K et al (2015) Relationship between choroidal blood flow velocity and choroidal thickness during systemic corticosteroid therapy for Vogt-Koyanagi-Harada disease. Graefes Arch Clin Exp Ophthalmol 253: 609-617

7. Goto K, Miki A, Araki S et al (2016) Time course of macular and peripapillary inner retinal thickness in non-arteritic anterior ischaemic optic neuropathy using spectral-domain optical coherence tomography. Neuroophthalmology 40: 74-85

8. Aizawa N, Yokoyama $\mathrm{Y}$, Chiba $\mathrm{N}$ et al (2011) Reproducibility of retinal circulation measurements obtained using laser speckle flowgraphy-NAVI in patients with glaucoma. Clin Ophthalmol 5: 11711176

9. Maekubo T, Chuman H, Nao-I N (2013) Laser speckle flowgraphy for differentiating between nonarteritic ischemic optic neuropathy and anterior optic neuritis. Jpn J Ophthalmol 57: 385-390

10. http://www.softcare-ltd.co.jp/

11. Riva CE, Titze P, Hero M, Petrig BL (1997) Effect of acute decreases of perfusion pressure on choroidal blood flow in humans. Invest Ophthalmol Vis Sci 38: 1752-1760

\section{Figures}



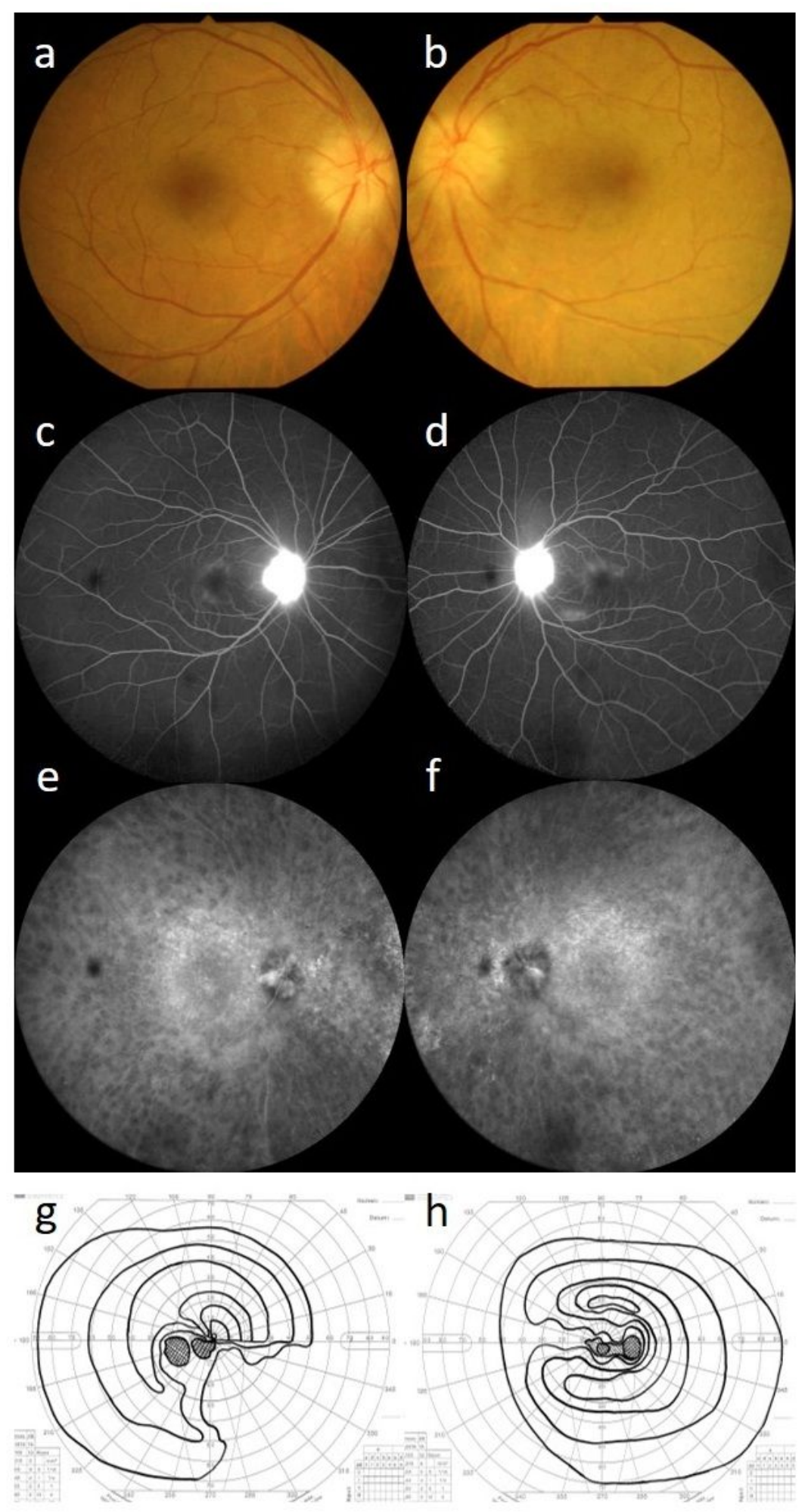

\section{Figure 1}

Photographs and Goldmann perimetry of the right eye $(a, c, e, h)$ and left eye $(b, d, f, g)$ at the initial visit. $a, b$ : Color fundus photos showing optic disc swelling in both eyes. c, d: Late-phase fluorescein angiography showing multifocal leakages in the posterior pole and optic disc staining. e, f: Indocyanine green angiography at the middle phase showing multiple hypofluorescent dark dots scattering all around 
the fundus. g, h: Bilateral visual field defects similar to inferior altitudinal hemianopsia, detected by Goldmann perimetry.
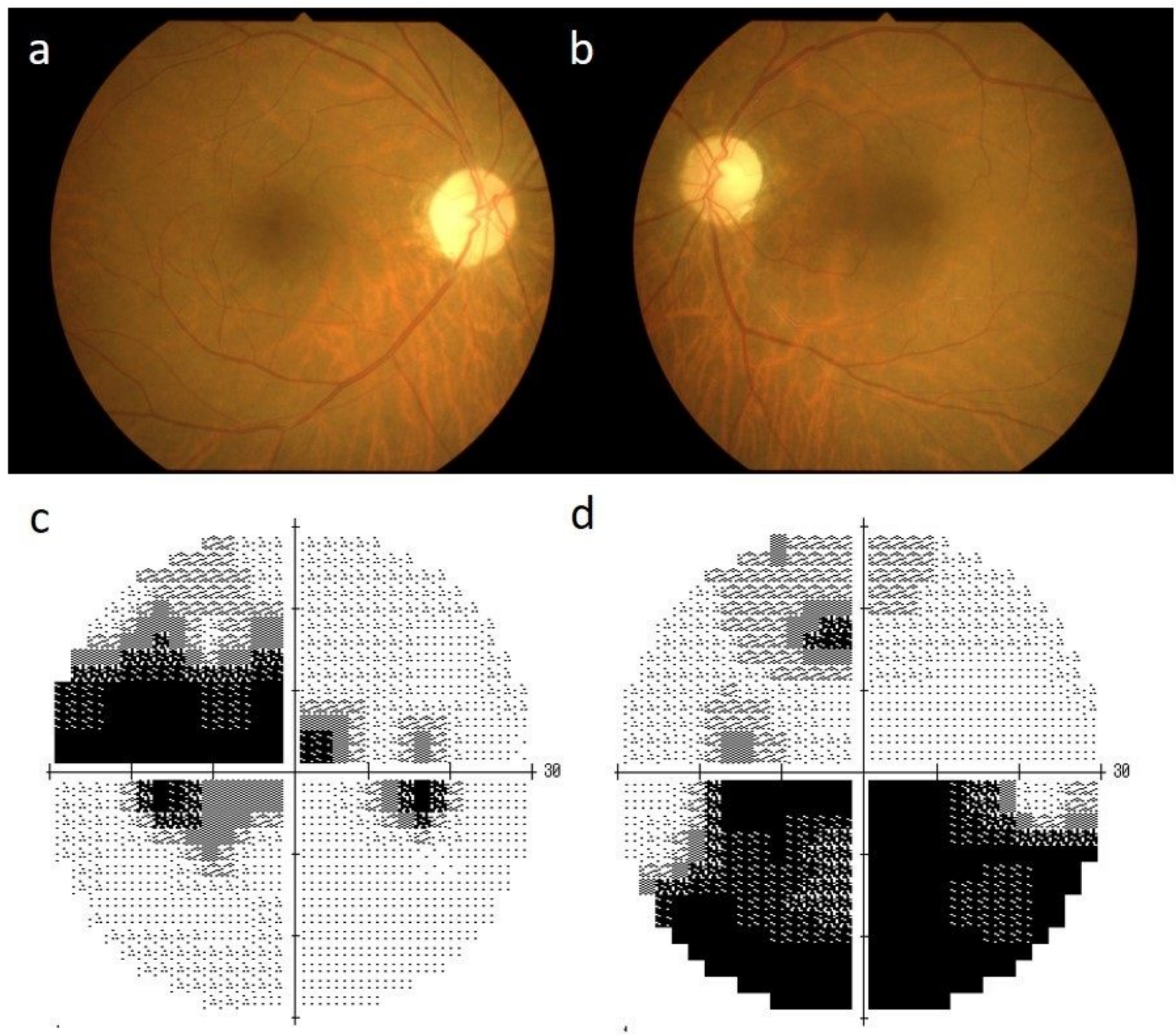

\section{Figure 2}

Photographs and Humphrey 30-2 visual fields at 5 months after the initiation of systemic corticosteroid therapy. Optic discs were observed to be slightly pale in both eyes (a: right eye; b: left eye). Visual field defects in both eyes still remained 5 months later (c: right eye; d: left eye). 


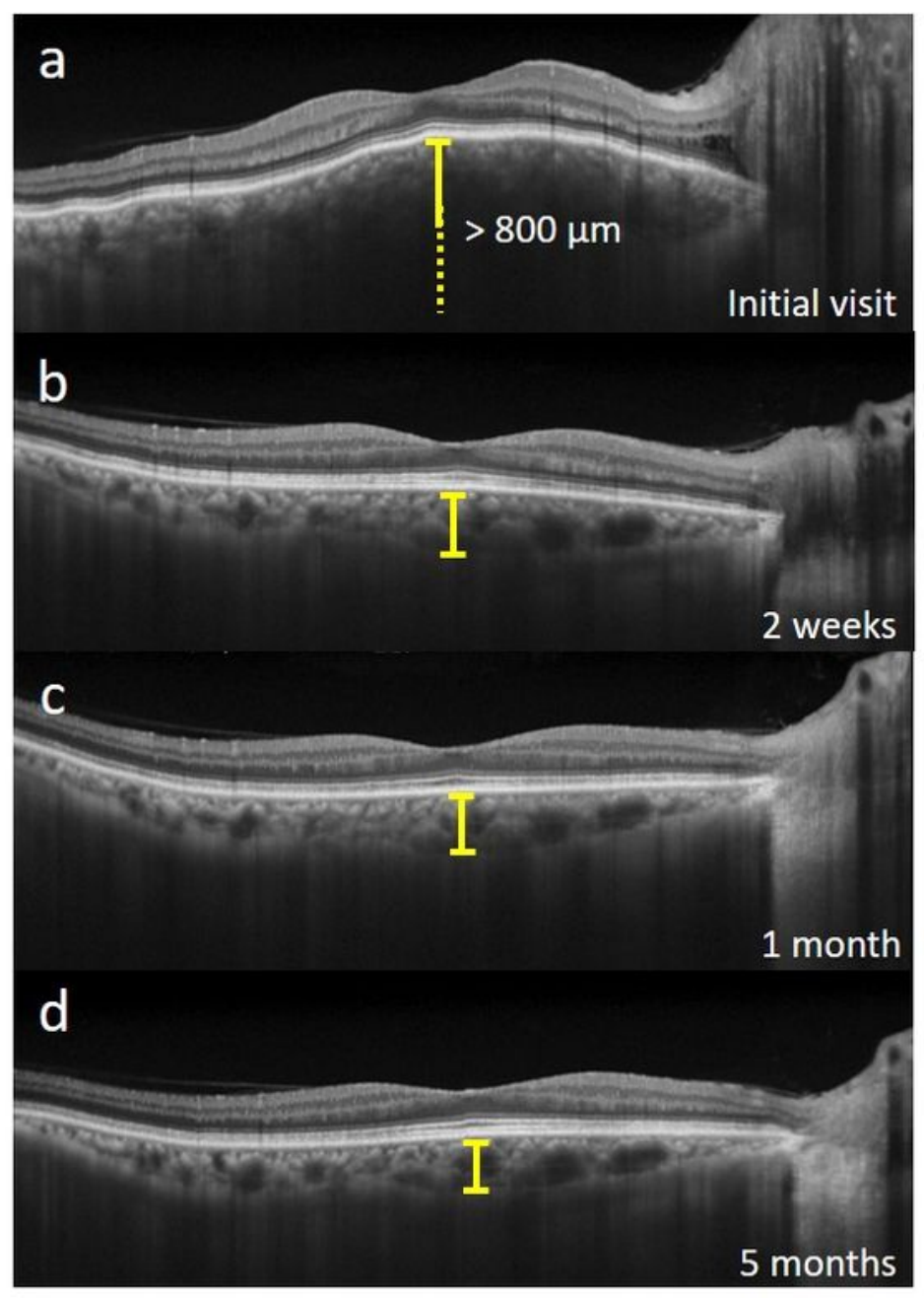

e

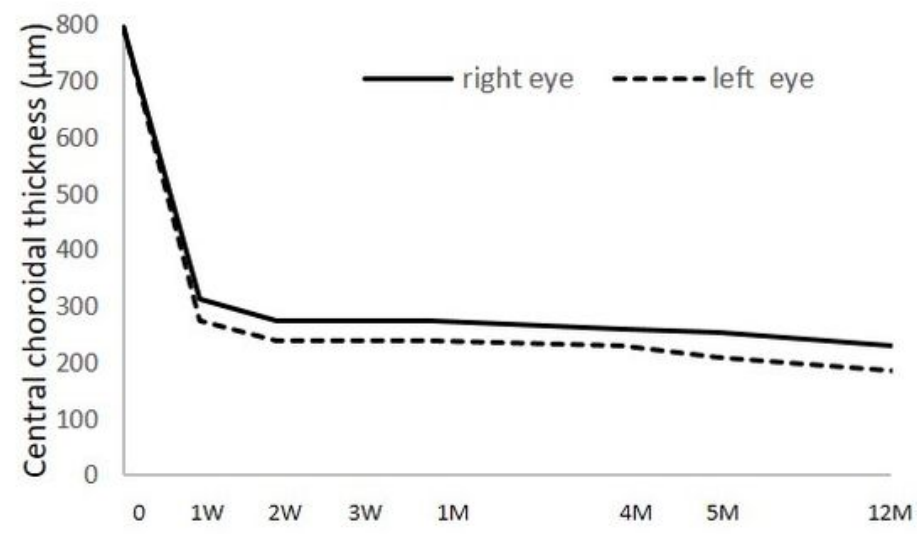

\section{Figure 3}

Horizontal images of enhanced depth optical coherence tomography (OCT) images across the fovea in the right eye during follow-up. Optic disc swelling was observed and central choroidal thickness (CCT) was $>800 \mu \mathrm{m}$ at the initial visit (a). The CCT decreased to $276 \mu \mathrm{m} 2$ weeks after the therapy (b); $276 \mu \mathrm{m}$ at 1 month (c); and $255 \mu \mathrm{m}$ at 5 months (d). Optic disc swelling also recovered with the treatment. E: A graph showing the time course of CCT changes in both eyes. 


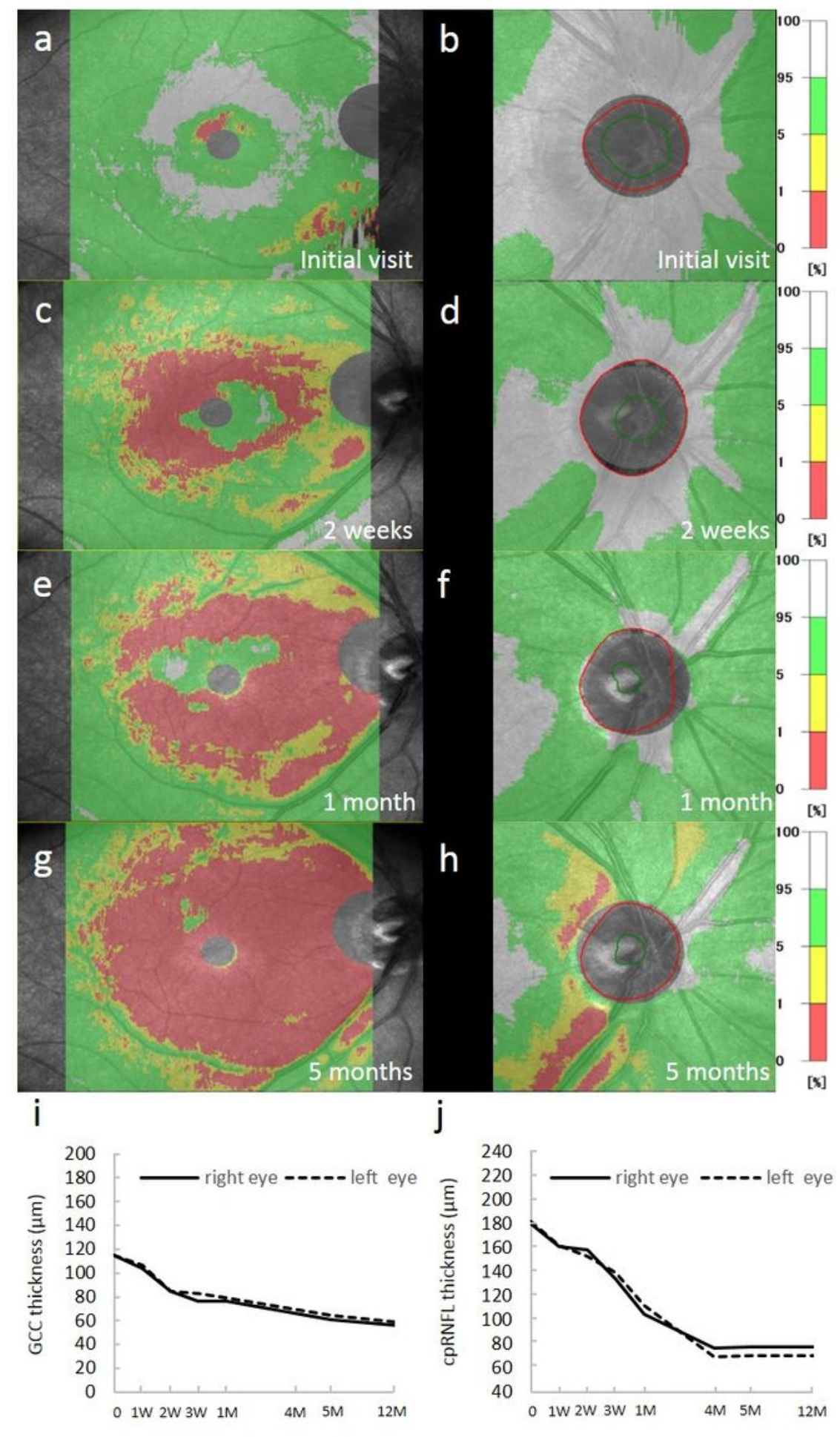

\section{Figure 4}

OCT C-scan images showing the ganglion cell complex (GCC) thickness map (a, c, e, g) and circumpapillary retinal nerve fiber layer (cpRNFL) thickness map $(b, d, f, h)$ in the right eye during followup. OCT provides thickness maps in which green indicates that the thickness is within the normal range; yellow and white indicate an increased abnormality below the $5 \%$ level; and red points to a decreased abnormality below the $1 \%$ level. The GCC thickness map indicated almost normal findings at the initial 
visit (a); the appearance of thinning area at 2 weeks later (c); a gradual spread over the whole macular area 1 month later; (e) and 5 months later (g). i shows the change of the GCC thickness in both eyes. The cpRNFL thickness map showed peripapillary RNFL thickening at the initial visit (b) and at week 2 (d). The thickness changed to almost normal at 1 month (f), then it showed thinning at 5 months (h). j shows the change of cpRNFL thickness in both eyes.

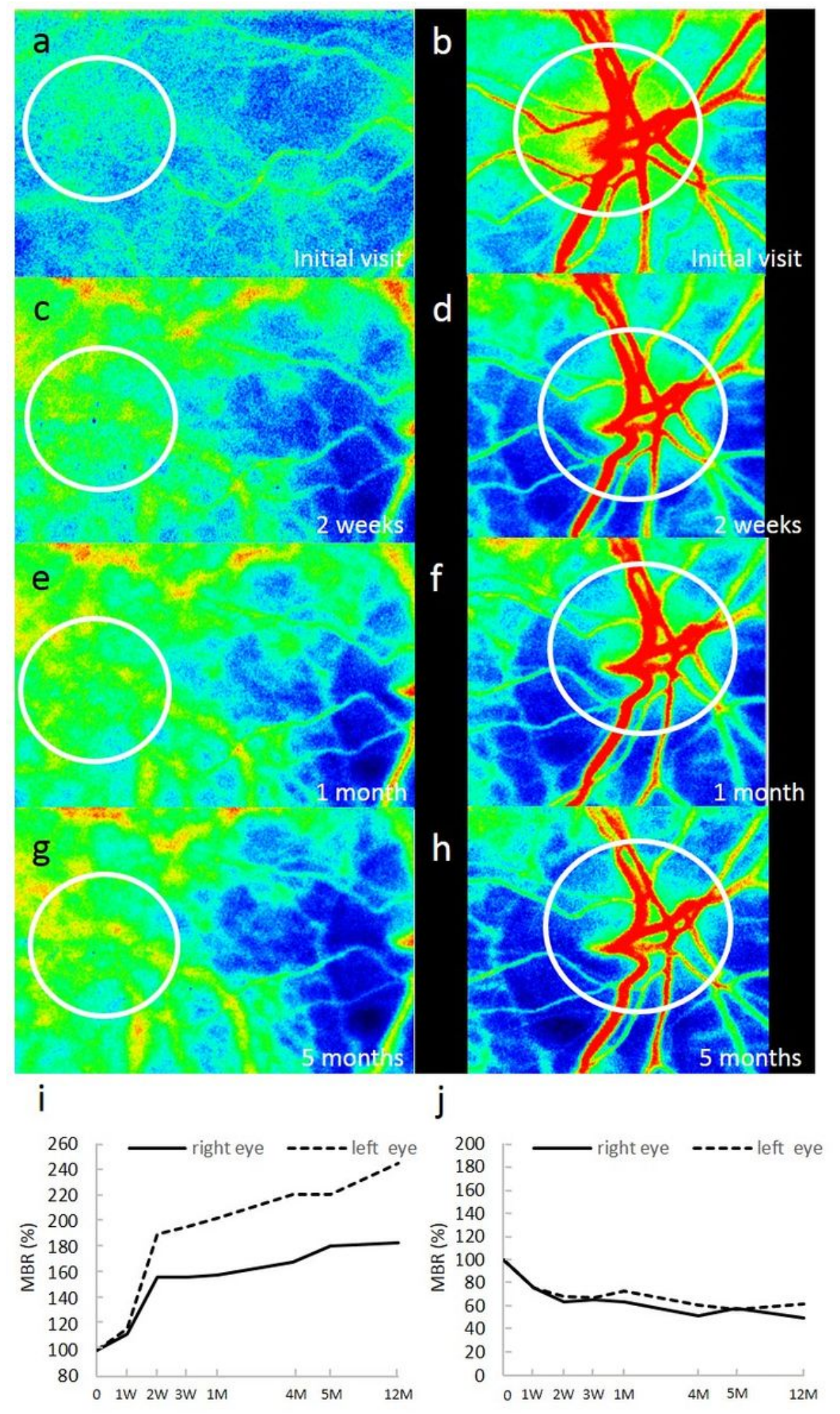

Figure 5 
Composite color map image of the mean blur rate (MBR) measured by laser speckle flowgraphy. Circles were set at the macula $(a, c, e, g)$ and optic nerve head $(\mathrm{ONH})(b, d, f, h)$. The blue color indicates low MBR, while the red color shows high MBR. The macular MBR increased at 2 weeks, 1 month, and 5 months (c, e, g), compared to that at baseline (a). i shows the change in the macular MBR in both eyes. The ONH tissue MBR decreased at 2 weeks, 1 month, and 5 months $(d, f, h)$ compared to that at baseline (b). j shows the change of the $\mathrm{ONH}$ tissue MBR in both eyes. 